\title{
A CHARACTERIZATION OF CONWAY'S GROUP $.3^{1}$
}

\author{
BY DANIEL FENDEL
}

Communicated by Murray Gerstenhaber, February 17, 1970

Statement of result. Let $G_{0}$ be the group .3 discovered by J. H. Conway [1], and let $s_{0}$ be an involution in the center of a Sylow 2 -subgroup of $G_{0}$. A direct examination of $G_{0}$ shows $\left(C_{G_{0}}\left(s_{0}\right) /\left\langle s_{0}\right\rangle\right.$ $\simeq \mathrm{Sp}(6,2)$, a nonsplit extension.

THEOREM. Let $G$ be a finite group and s an involution in $G$, such that $C_{G}(s) \simeq C_{G_{0}}\left(s_{0}\right)$. Assume $G \neq C_{G}(s) O(G)(O(G)$ is the maximal normal odd order subgroup of $G$ ). Then $G \simeq G_{0}$. In particular, $G$ has the following properties:

(i) G has order $2^{10} \cdot 3^{7} \cdot 5^{3} \cdot 7 \cdot 11 \cdot 23$, and is simple.

(ii) $G$ has two conjugacy classes of involutions. One class is represented by the involution $s$. A representative $t$ of the second class has centralizer $C_{G}(t) \simeq\langle t\rangle \times M_{12}$ ( $M_{12}$ is the Mathieu group).

(iii) The normalizer of a Sylow 23-subgroup is a Frobenius group of order $11 \cdot 23$.

(iv) The normalizer of a Sylow 11-subgroup is a direct product of $Z_{2}$ (the group of order 2) and a Frobenius group of order $5 \cdot 11$.

(v) The normalizer of a Sylow 7-subgroup is a direct product of $\mathrm{Sym}_{3}$ (the symmetric group) and a Frobenius group of order $6 \cdot 7$ with kernel of order 7.

(vi) A Sylow 5-subgroup is nonabelian of exponent 5. There are two classes of elements of order 5 , with centralizers of orders $2^{2} \cdot 3 \cdot 5^{3}$ and $2^{2} \cdot 3 \cdot 5^{2}$. The normalizer of a Sylow 5-subgroup has order $2^{4} \cdot 3 \cdot 5^{3}$.

(vii) $G$ has no outer automorphisms.

The character table of $G$ is obtained in the course of the proof, and will appear elsewhere with details of the proof.

Outline of proof. Property (ii) is proved using group-theoretic methods and Wong's characterization of $M_{12}$ [2]. The order follows using a formula of Thompson's requiring only knowledge of the centralizers of involutions and conjugacy of involutions. Properties (iii)-(vi) are then straightforward. This provides sufficient informa-

AMS 1968 subject classifications. Primary 2027, 2029; Secondary 2020, 2022, 2060, 2080.

Key words and phrases. Conway's group .3, characterization of simple group, centralizer of involution, character table, Leech lattice.

1 This research is contained in the author's doctoral dissertation submitted to Yale University, and was supported by a National Science Foundation Graduate Fellowship. The author wishes to thank his advisor, Professor, Walter Feit, for his interest and assistance throughout this work. 
tion to determine the character table, with the aid of a computer. The most important techniques used are these:

(i) The $p$-block structure for $p=23,11$, and 7 .

(ii) Equations involving the values of characters on involutions, expressing the nonrealness of certain classes.

(iii) Orthogonality relations.

In particular, the group is seen to have a rational, absolutely irreducible character of degree 23 . We then use the following result due to Feit: if a group $H$ has a rational, absolutely irreducible, faithful representation of degree 23 , and no subgroup of index 23 or 24 , then $H$ acts on one of three 23-dimensional lattices [3]. These lattices can be found as sublattices of the Leech lattice, and are the orthogonal complements, respectively, of vectors of types 2,3 , and $6_{3,2}$ (see [1]). The automorphism groups of these lattices are determined to be, respectively, $.2 \times Z_{2}, .3 \times Z_{2}$, and $M_{24} \times Z_{2}$. The orders of these groups now provides the conclusion $G \simeq G_{0}$. Property (vii) follows from the corresponding fact for $\operatorname{Sp}(6,2)$ and the characterization of $G_{0}$.

Some remarks on the characters of $G_{0}$. $G_{0}$ has 42 irreducible characters. The degrees are as follows: $1,23,253,253,275,896,896,1771$, 2024, 3520, 3520, 4025, 5544, 7084, 8855, 9625, 9625, 20608, 20608, $23000,26082,31625,31625,31625,31878,40250,57960,63250,73600$, $80960,91125,93312,129536,129536,177100,184437,221375,226688$, $246400,249480,253000,255024$. The characters of degrees 896,3520 , 9625 , and 20608 occur in complex-conjugate pairs; the remaining 34 characters are rational.

For $p=23,11$, and 7, the blocks are as described by Brauer [4]. For $p=5$, there is the principal block, one block of defect 1 , and blocks of defect 0 . For $p=3$, there is the principal block and one block of defect 1 ; there are no 3 -blocks of defect 0 . For $p=2$, there is the principal block, one block of defect 1 , and one block of defect 3 (which has 8 characters, all of height 0 , and an elementary abelian defect group); there are no 2-blocks of defect 0 .

\section{REFERENCES}

1. J. H. Conway, $A$ perfect group of order $8,315,553,613,086,720,000$ and the sporadic simple groups, Proc. Nat. Acad. Sci. U.S.A. 61 (1968), 398-400. MR 38 \#5915.

2. W. J. Wong, $A$ characterization of the Mathieu group $M_{12}$, Math. Z. 84 (1964), 378-388. MR 29 \#4794.

3. Private communication.

4. R. Brauer, On groups whose order contains a prime number to the first power. I, Amer. J. Math. 64 (1942), 401-420. MR 4, 1.

Yale University, New Haven, Connecticut 06520 